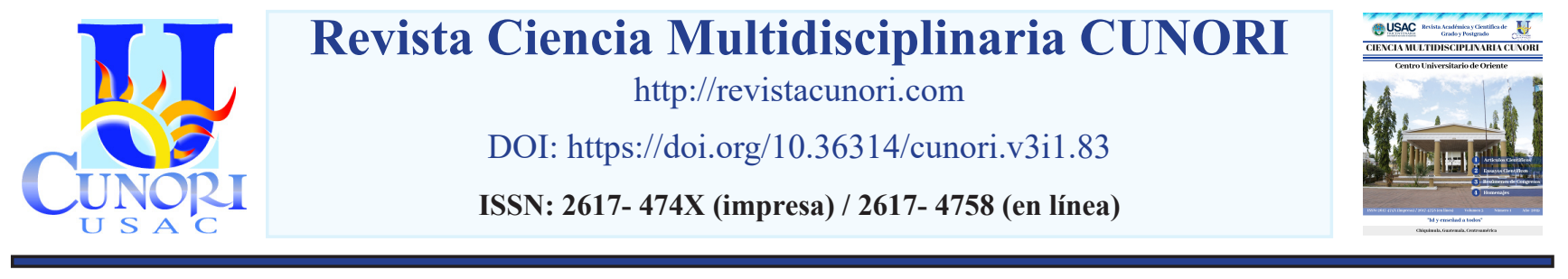

Como citar el artículo

Villeda, S., Martínez, C., Duarte, K., Retana, R., Arriola, C., Mazariegos, E., (2019). Características microbiológicas de sepsis neonatal. Revista Ciencia Multidisciplinaria CUNORI, 3(1), 85-92. DOI: https://doi.org/10.36314/cunori.v3i1.83

\title{
Características microbiológicas de sepsis neonatal
}

\section{Microbiological characteristics of neonatal sepsis}

\author{
Sandra Villeda*, Celia Martínez, Karem Duarte, Ronaldo Retana, Carlos Arriola \\ Centro Universitario de Oriente (CUNORI), Universidad de San Carlos de Guatemala
}

Recibido: 30 de agosto de 2018 / Revisión: 28 de septiembre de 2018 / Aceptado: 21 de enero de 2019

Disponible en internet el 30 de agosto de 2019

*Autor para correspondencia.

Resumen

Correo electrónico: sandrita.vs41@gmail.com

Qepsis neonatal es un síndrome clínico caracterizado por signos sistémicos de infección, ocasionados por la invasión de agentes $\checkmark$ patógenos dentro del torrente sanguíneo. El aislamiento de bacterias mediante el hemocultivo es el estándar de oro para confirmar su diagnóstico. El diagnóstico debe ser precoz y el tratamiento debe iniciarse antes de tener resultados de hemocultivo basándose en los microorganismos más comunes de la localidad. El Hospital Nacional de Chiquimula no siempre cuenta con el recurso para la realización de hemocultivo a los pacientes, por lo que se desconoce el perfil microbiológico y patrones de resistencia local, por lo cual se decidió realizar un estudio descriptivo transversal en los meses de junio y julio del año 2018 con el fin de determinar las características microbiológicas de sepsis neonatal, su patrón de sensibilidad y resistencia antimicrobiana, tomando en cuenta 80 pacientes con diagnóstico de sepsis neonatal o sospecha de sepsis neonatal a quienes se les realizó un hemocultivo antes de iniciar tratamiento antibiótico. El diagnóstico de los pacientes se realizó basado en los antecedentes maternos y las alteraciones hematológicas del paciente. Los 80 hemocultivos fueron negativos a los siete días de incubación por lo que lo que no fue posible conocer los microorganismos causantes de sepsis neonatal, sensibilidad y resistencia antimicrobiana, sin embargo se observó mejoría en el $81 \%$ de los pacientes con el tratamiento antibiótico empírico utilizado, se logró comprobar que el tratamiento empírico utilizado en el Hospital Nacional de Chiquimula continua siendo eficaz en los pacientes con sepsis neonatal.

Palabras clave: sepsis neonatal, hemocultivo, sensibilidad, resistencia

Abstract

Teonatal sepsis is a clinical syndrome characterized by systemic signs of infection, caused by the invasion of pathogens into 1 the bloodstream. Isolation of bacteria by blood culture is the gold standard to confirm your diagnosis. The diagnosis must be early and the treatment must be started before having blood culture results based on the most common microorganisms in the locality. The National Hospital of Chiquimula does not always have the resource to carry out blood culture to patients, so the microbiological profile and local resistance patterns are unknown, so it was decided to carry out a cross-sectional descriptive study in the months of June and July 2018 in order to determine the microbiological characteristics of neonatal sepsis its pattern of sensitivity and antimicrobial resistance, taking into account 80 patients diagnosed with neonatal sepsis or suspected neonatal sepsis who underwent a blood culture before starting antibiotic treatment. The diagnosis of the patients was made based on the maternal history and the haematological alterations of the patient. The 80 blood cultures were negative at seven days of incubation, so it was not possible to know the microorganisms causing neonatal sepsis, sensitivity and antimicrobial resistance, however improvement was observed in $81 \%$ of the patients with the empirical antibiotic treatment used, it was verified that the empirical treatment used in the National Hospital of Chiquimula continues to be effective in patients with neonatal sepsis.

Keywords: neonatal sepsis, blood culture, sensitivity, resistance 


\section{Introducción}

Las infecciones neonatales provocan alrededor de 1.6 millones de muertes, en su mayoría debido a sepsis y meningitis. En América latina la incidencia de sepsis neonatal se encuentra en 3.5 y 8.9 por ciento (Coronell, Perez, Guerrero \& Bustamante 2009:59). Se entiende por sepsis neonatal aquella situación clínica derivada de la invasión y proliferación de bacterias, hongos o virus en el torrente sanguíneo del recién nacido $(\mathrm{RN})$ y que se manifiesta en los primeros 28 días de vida.

Los microorganismos patógenos inicialmente contaminan la piel y/o mucosas del recién nacido llegando al torrente circulatorio tras atravesar esta barrera, siendo la inmadurez de las defensas del neonato, el principal factor de riesgo que predispone al desarrollo de la sepsis (AEP 2008:192).

El diagnóstico de sepsis neonatal debe ser precoz y el tratamiento debe iniciarse antes de tener resultados de hemocultivo basándose en los microorganismos más comunes de la localidad. El Hospital Nacional de Chiquimula no siempre cuenta con el recurso para la realización de hemocultivo a los pacientes, por lo que se desconoce el perfil microbiológico y patrones de resistencia local, por lo cual se decidió realizar un estudio descriptivo transversal con el fin de determinar las características microbiológicas de sepsis neonatal en nuestra localidad. Se determinó que el $81 \%$ de los pacientes mejoraron 48 horas después de iniciar el tratamiento empírico.

\section{Material y métodos}

Se realizó un estudio descriptivo transversal sobre las características microbiológicas de sepsis neonatal en pacientes con diagnóstico de sepsis neonatal o sospecha de sepsis neonatal ingresados a los servicios de neonatología y pediatría del Hospital Nacional de Chiquimula en los meses de junio y julio del año 2018. Se estudiaron 80 pacientes con diagnóstico de sepsis o sospecha de sepsis neonatal a quienes no se les había iniciado tratamiento antibiótico.

Se realizó el registro de datos de los pacientes por medio de una boleta de recolección de datos que constaba de los siguientes apartados: Datos generales del paciente: No. de boleta, sexo y edad. Datos clínicos: Tipo de parto y diagnóstico. Datos de laboratorio: Hemograma de ingreso. Resultados de hemocultivo: Microorganismo aislado, sensibilidad y resistencia. Se realizó un hemocultivo tomando 1 $\mathrm{ml}$ de sangre extraída por venopunción de vía periférica la cual fué sembrada en tres agares diferentes, MacConkey, chocolate y sangre, por siete días para observar crecimiento bacteriano. 


\section{Resultados}

Distribución de resultados de hemocultivos en neonatos

\begin{tabular}{|c|c|c|}
\hline $\begin{array}{c}\text { Cultivos } \\
\text { positivos }\end{array}$ & $\begin{array}{c}\text { Cultivos } \\
\text { Negativos }\end{array}$ & $\begin{array}{c}\text { Gérmenes } \\
\text { Aislados }\end{array}$ \\
\hline 0 & 80 & Ninguno \\
\hline
\end{tabular}

Cuadro 1: Distribución de resultados de hemocultivos en neonatos con diagnóstico de sepsis neonatal en el Hospital Nacional de Chiquimula

Se observa el reporte de resultado de hemocultivo realizado en pacientes con diagnóstico de sepsis, en la cual se observa que el 100\%(80) fueron negativos a los siete días de incubación.

Distribución de pacientes con diagnóstico de sepsis

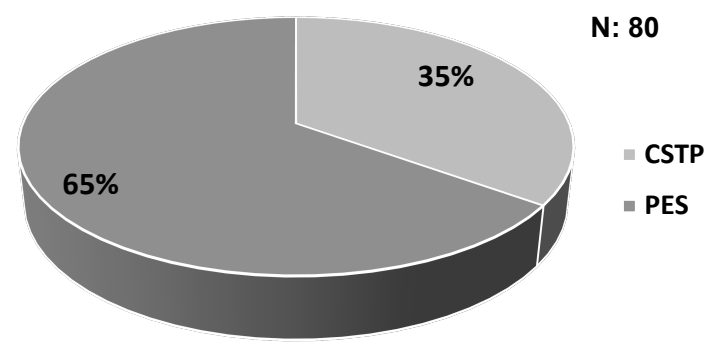

Figura 1. Distribución de pacientes con diagnóstico de sepsis según el tipo de parto en el Hospital Nacional de Chiquimula, durante junio y julio de 2018.

El 65\% (52) de los pacientes incluidos en el estudio fueron producto de parto eutócico simple, en comparación del 35\% (28) que nacieron por cesárea.

Distribución de pacientes con diagnóstico de sepsis

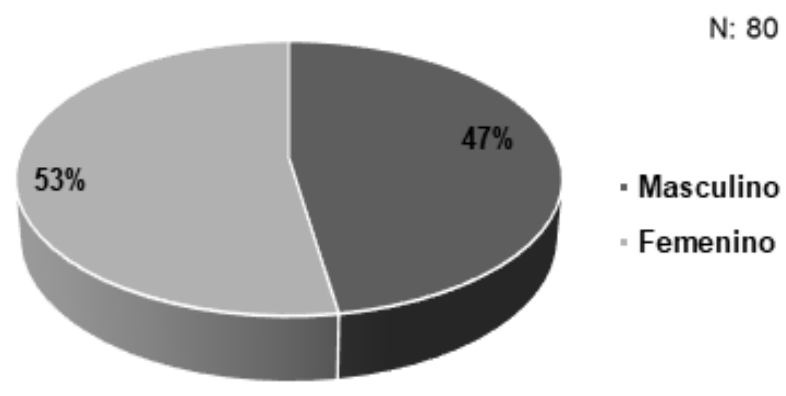

Figura 2. Distribución de pacientes con diagnóstico de sepsis neonatal según el sexo en el Hospital Nacional de Chiquimula, durante junio y julio de 2018

En esta gráfica se observa que el 53\% (42) de los pacientes, fueron de sexo femenino y el 47\% (38) de los pacientes que fueron sexo masculino. 
Distribución de pacientes según el tiempo que transcurrió después del nacimiento

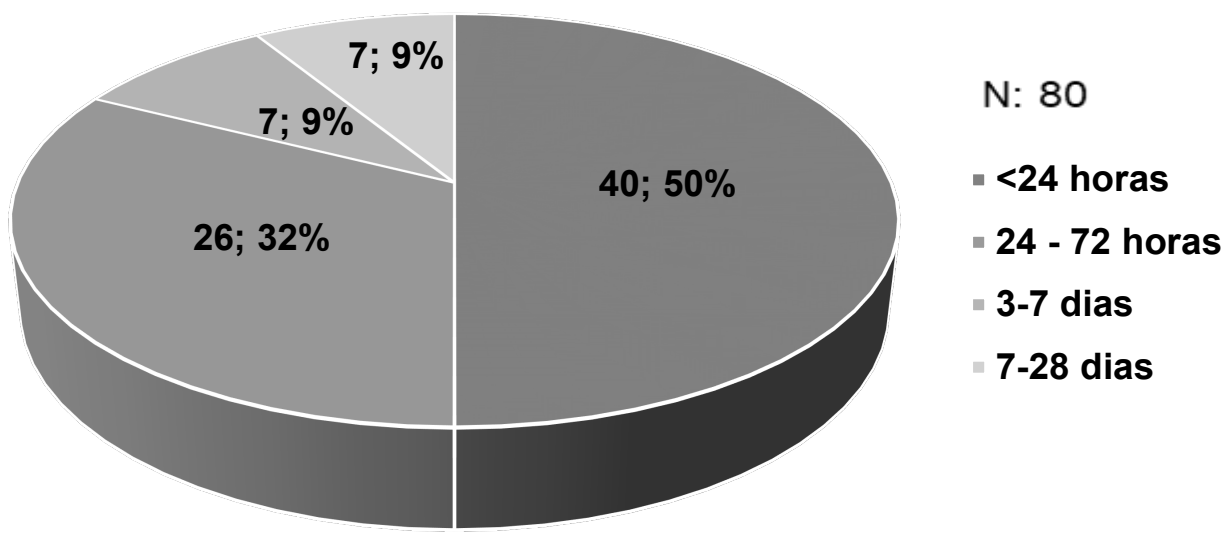

Figura 3. Distribución de pacientes según el tiempo que transcurrió después del nacimiento en el cual se realizó el diagnóstico de sepsis neonatal en el Hospital Nacional de Chiquimula en los meses de junio y julio de 2018

El 40.5\% (40) de los pacientes ingresaron con diagnóstico de sepsis neonatal en un tiempo menor de 24 horas después del nacimiento, el 26.32\% (26) en un rango de 24-72 horas después del nacimiento, $7.9 \%$ (7) de los pacientes en un rango de 3-7 días y 7.9\% (7) en rango de 7-28 días.

\section{Distribución de pacientes según el diagnóstico de ingreso}

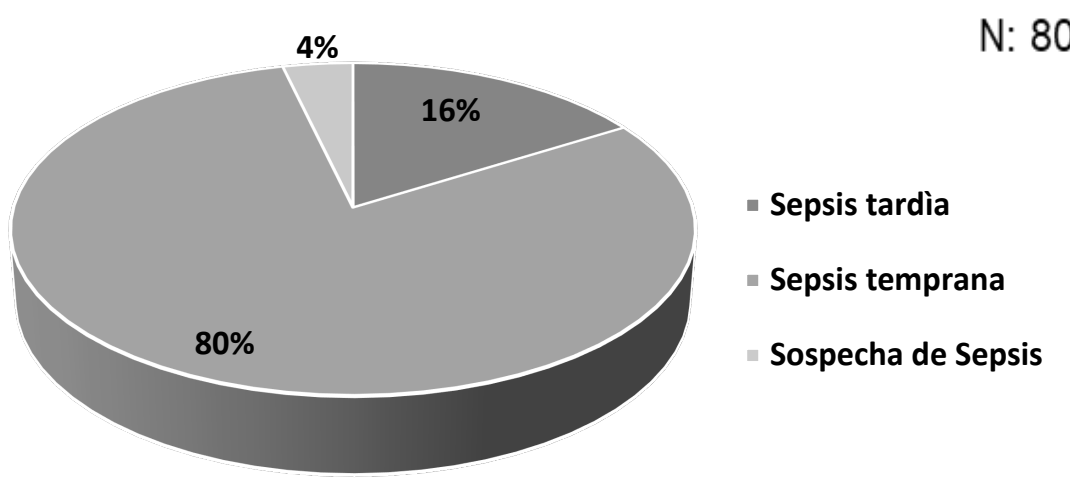

Figura 4. Distribución de pacientes según el diagnóstico de ingreso en los servicios de neonatología y pediatría del Hospital Nacional de Chiquimula en los meses junio y julio 2018.

El 80\% (64) de los pacientes fue ingresado con diagnóstico de sepsis temprana, 16\% (13) con diagnóstico de sepsis tardía y solo un $4 \%$ (3) con diagnóstico de sospecha de sepsis. 
Distribución de pacientes con diagnóstico de sepsis neonatal según conteo de glóbulos blancos

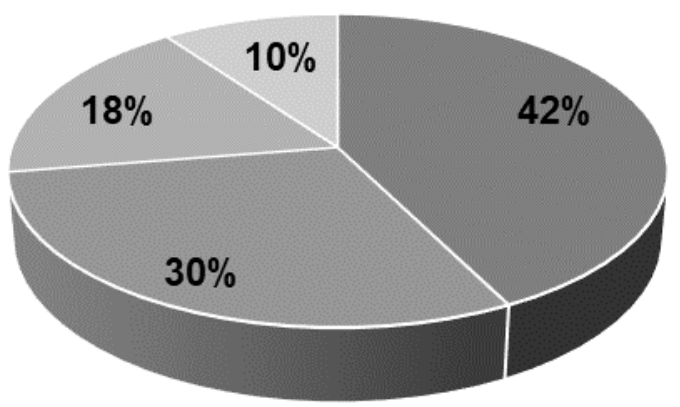

$\mathrm{N}: 80$

- $6 \mathrm{mil}-20 \mathrm{mil}$

$=20 \mathrm{mil}-25 \mathrm{mil}$

$=25 \mathrm{mil}-30 \mathrm{mil}$

- $>30 \mathrm{mil}$

Figura 5. Distribución de pacientes con diagnóstico de sepsis neonatal según conteo de glóbulos blancos en hematología de ingreso en el Hospital Nacional de Chiquimula en los meses de junio y julio 2018

El 42\% (34) de los pacientes ingresados por sepsis neonatal, presentaron leucocitos en un rango de 6 mil a 20 mil, un 30\%(24) de 20 a 25 mil, 18\% (14) de 25 a 30 mil y un 10\% (8) mayor de 30 mil leucocitos.

Distribución de pacientes con diagnóstico de sepsis neonatal según conteo de neutrófilos

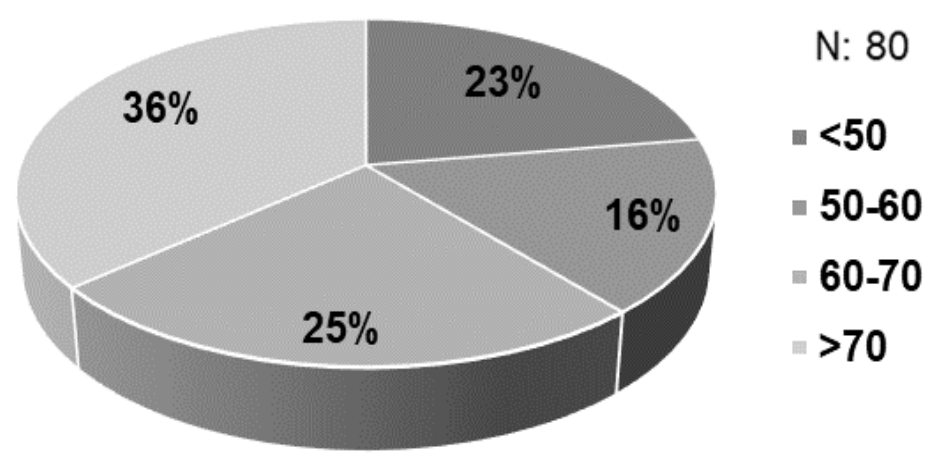

Figura 6. Distribución de pacientes con diagnóstico de sepsis neonatal según conteo de neutrófilos en hematología de ingreso en el Hospital Nacional de Chiquimula en los meses de junio y julio 2018

Del total de pacientes ingresados, 36\% (29) presentaron un valor mayor del $70 \%$ de neutrófilos, el $25 \%$ (20) de los pacientes presentaron valores en un rango de 60-70\%, el 23\%(18) presentaron menos del $50 \%$ de neutrófilos y el $16 \%(13)$ en un rango de $50-60 \%$ de neutrófilos en la hematología de ingreso. 
Distribución de pacientes con diagnóstico de sepsis neonatal según los valores de plaquetas

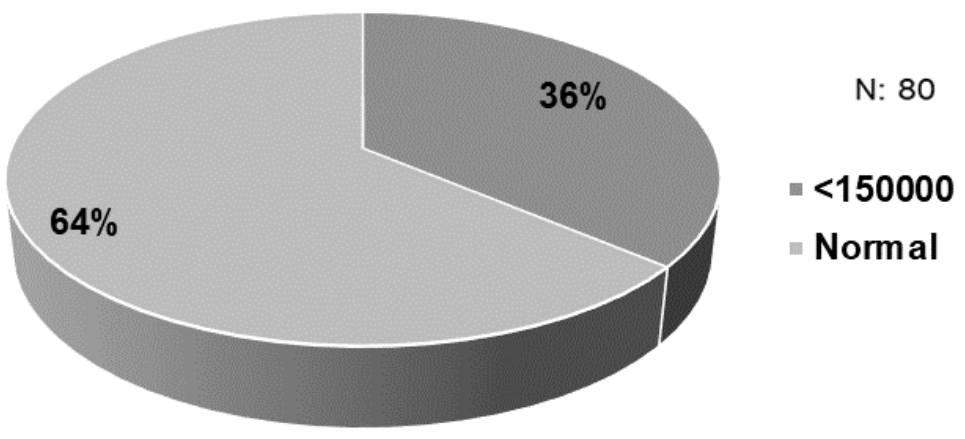

Figura 7. Distribución de pacientes con diagnóstico de sepsis neonatal según los valores de plaquetas en hematología de ingreso en el Hospital Nacional de Chiquimula en los meses de junio y julio del 2018.

Del total de pacientes ingresados, 64\% (51) presentaron conteo normal de plaquetas en la hematología y $36 \%(29)$ presentó trombocitopenia al momento del ingreso.

Distribución de pacientes con diagnóstico de sepsis neonatal según su evolución clínica

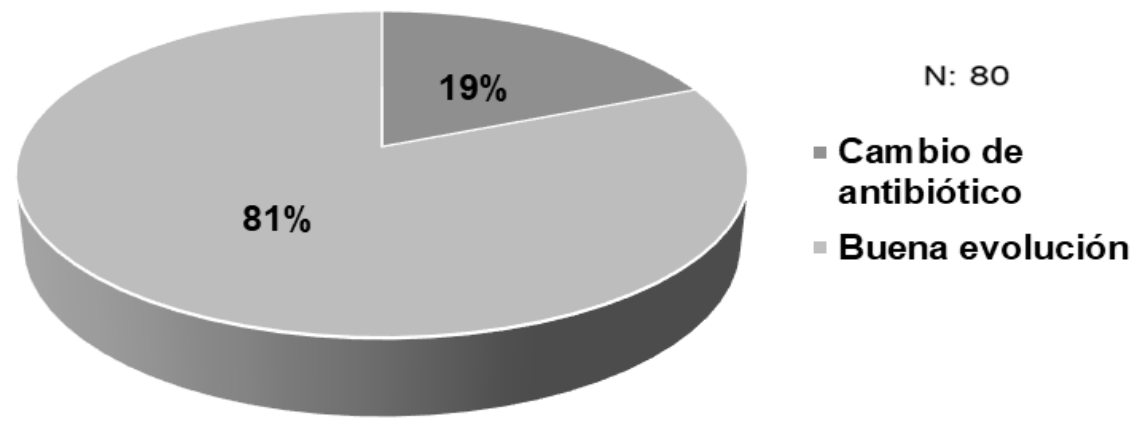

Figura 8. Distribución de pacientes con diagnóstico de sepsis neonatal según su evolución clínica con el tratamiento antibiótico empírico.

En esta gráfica se observa que el $81 \%$ (65) de los pacientes presentó buena evolución con el tratamiento antibiótico empírico, y solo un 19\% (15) presentó mala evolución clínica.

\section{Resultados}

De los hemocultivos realizados, el $100 \%$ resultaron estériles a los siete días de incubación, por lo que no fue posible conocer los microorganismos más comunes causantes de sepsis neonatal en el Hospital Nacional de Chiquimula y tampoco se pudo determinar la sensibilidad y resistencia a los antimicrobianos.

Uno de los principales problemas clínicos en la sepsis neonatal es la dificultad para establecer cuándo realmente está presente, tal como se ha informado en estudios previos en los que se tiene una alta frecuencia de recién nacidos con datos clínicos sugestivos de sepsis, con signos de respuesta inflamatoria 
sistémica, sin confirmación microbiológica por aislamiento (Marín, Leboreiro, Bernárdez \& Rendón 2015:95). Con el fin de garantizar que todos los recién nacidos infectados sean identificados y tomando en cuenta que no se poseen todas las pruebas diagnósticas necesarias, los criterios para diagnóstico de sepsis neonatal en el Hospital de Chiquimula suelen ser muy amplios, lo que puede llevar a diagnosticar y tratar bebés no infectados.

En ocasiones por temor a un deterioro del recién nacido, y tomando en cuenta todos los factores a los que están expuestos los recién nacidos en nuestro entorno como lo son factores culturales por parte de la madre, mal control o incluso ausencia de control prenatal y difícil acceso a los servicios de salud, se decide clasificar como sepsis a un episodio, e iniciar antibiótico sin tener un diagnóstico confirmado, esto es aceptable por la alta vulnerabilidad de estos pacientes. Cada sospecha de sepsis debe ser evaluada para definir qué tan sólido es el diagnóstico y decidir la continuidad o suspensión de antibióticos administrados recordando que la decisión de interrumpir el tratamiento antibiótico debe incluir la evaluación de los resultados de otras pruebas de laboratorio utilizadas para sepsis y que no solo debe basarse en un hemocultivo negativo.

El hemocultivo continúa siendo el estándar de oro para el diagnóstico de sepsis, y su positividad establece el diagnóstico definitivo de sepsis neonatal, sin embargo su tasa de positividad es baja, esto puede explicarse en parte por tratamientos antimicrobianos administrados a la madre, conteos de colonias bacterianas $\leq 4 \mathrm{UFC} / \mathrm{mL}$ y al poco volumen de sangre extraído. Utilizando un volumen de sangre de $1 \mathrm{ml}$ la sensibilidad es del 30 al $40 \%$, usando un volumen de $3 \mathrm{ml}$ la sensibilidad aumenta hasta un 70 a $80 \%$, durante la investigación la muestra extraída fue de $1 \mathrm{ml}$ de sangre.

Se habla de una sepsis probable cuando no es posible aislar un patógeno en un hemocultivo, pero el recién nacido tiene un curso clínico sugestivo de sepsis neonatal y anomalías hematológicas que sugieren sepsis; en las cuales se sugiere realizar pruebas seriales de laboratorio con el fin de identificar a todos los recién nacidos infectados (Morven 2017:13). Al 100\% de la muestra se inició tratamiento antibiótico de primera línea, utilizando la combinación de ampicilina y un aminoglucósido (amikacina), siendo éste el esquema antibiótico empírico para el manejo de la sepsis neonatal basándose en la epidemiología de los agentes etiológicos más probablemente involucrados según la literatura, con dicho tratamiento se observó mejoría del 81\% de los pacientes quienes fueron egresados a las 48 horas, y un 19\% presentó evolución clínica o hematológica desfavorable por lo que necesitaron más días de ingreso hospitalario, realizar otros cultivos y cambio de cobertura antibiótica.

Por lo que en este hospital se sigue considerando efectivo el uso de ampicilina y un aminoglucósido como el esquema empírico inicial de antimicrobianos. Según el tipo de parto, del total de la muestra $65 \%$ nació por parto eutócico simple y el 35\% por cesárea. Se observó un predominio del sexo femenino $53 \%$ en comparación con el $47 \%$ que fueron de sexo masculino. En el perfil hematológico de ingreso, de los 80 pacientes, el 42\% no presentó alteración leucocitaria, 30\% presentaron un recuento dentro del rango de 20 mil a 25 mil leucocitos/ mm3, 18\% de 25 mil a 30 mil leucocitos/ mm3 y solamente el $10 \%$ presentó leucocitos mayor de 30 mil. Según el conteo de neutrófilos, el 36\% presentó un recuento de neutrófilos mayor al 70\%, un 25\% se encontraron en un rango de 60-70\%, el 23\% presentó valores menores al 50\% y el 16\% en un rango de 50-60\%. En el recuento de plaquetas, $64 \%$ mostraron conteo de plaquetas normales y el $36 \%$ presentaron trombocitopenia $(<150,000)$. 


\section{Agradecimientos}

Al Hospital Nacional de Chiquimula por permitirnos realizar nuestra investigación. A la Dra. Karem del Rosario Duarte especialista en pediatría, agradecemos su asesoría y revisión del nuestro trabajo. A las autoridades del Centro universitario de oriente, Dr. Edvin Danilo Mazariegos y Dr. Carlos Arriola por su guía y apoyo durante la realización de nuestra investigación.

\section{Referencias bibliográficas}

Coronell, W; Pérez, C; Guerrero, C; Bustamante, H. 2009. Sepsis neonatal (en línea). Revista de Enfermedades Infecciosas en Pediatría 23(90):57-68. Consultado 11 feb. 2018. Disponible en http:// www.medigraphic.com/pdfs/revenfinfped/eip-2009/eip094f.pdf

Marín-Romero M, Iglesias-Leboreiro J, BernárdezZapata I, Rendón-Macías ME. Características de la sepsis neonatal en una Unidad de Cuidados Intensivos Neonatales de atención privada. Rev Mex Pediatr 2015; 82(3):93-97 [Characteristics of neonatal sepsis in a Neonatal Intensive Care Unit of private care] Consultado 17 feb. 2018. Disponible en http:/www.medigraphic.com/pdfs/pediat/sp2015/sp153c.pdf

Morven S, E. 2017. Clinical features, evaluation, and diagnosis of sepsis in term and late preterm infantes (en línea, sitio web). UpToDate. p. 1-19. Consultado 03 jun. 2018. Disponible en https:/www.update.com/contents/clinical-features-evaluation-and-diagnosis-of-sepsis-in-term-and-late-preterm-infants? search=MICROBIOLOGIA $\% 20$ EN $\% 20$ SEPSIS $\% 20$ NEONATAL\&source=search_result\&selectedTitle=1 93\&usage_type=default\&display_rank=1

\section{Sobre la autora}

\section{Sandra Maribel Villeda Salguero}

Médico y Cirujano, egresada del Centro Universitario de Oriente CUNORI de la Universidad de San Carlos de Guatemala. Ha realizado investigaciones sobre "Papanicolaou más la prueba de tamizaje inspección visual con ácido acético (IVAA) para el diagnóstico temprano de cáncer de cérvix”, y la tesis que tiene como título "Características microbiológicas de sepsis neonatal”.

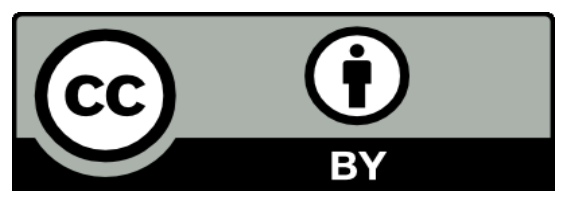

Este texto está protegido por una licencia CreativeCommons 4.0.

Usted es libre para compartir, copiar y redistribuir el material en cualquier medio o formato y adaptar el documento, remezclar, transformar y crear a partir del material para cualquier propósito, incluso comercialmente, siempre que cumpla la condición de atribución: usted debe reconocer el crédito de una obra de manera adecuada, proporcionar un enlace a la licencia, e indicar si se han realizado cambios. Puede hacerlo en cualquier forma razonable, pero no de forma tal que sugiera que tiene el apoyo del licenciante o lo recibe por el uso que hace. 